\title{
Critérios diagnóstico e métodos de atualização em diabetes gestacional segundo ginecologistas obstétricos da cidade de Araguari - MG
}

DOI: 10.47224/rm.v5i10.91

\author{
Lara Amélia Queiroz \\ Iara Guimarães Rodrigues \\ Ana Helena Bittencourt Alamy \\ Thaís Ribeiro Oliveira Santos de Marcello \\ Nathália Nunes Bessa Sousa \\ Brathener Paulo Moura Araújo \\ Nataska Batista Possas \\ Danilo Martins Júnior
}

e-mail: laraameliaq@gmail.com

\begin{abstract}
Resumo
Diabetes mellitus gestacional (DMG) é qualquer grau de redução da tolerância à glicose, resultando em hiperglicemia de gravidade variável, cuja deteç̧ão ocorreu durante a gravidez. O DMG é um distúrbio da segunda metade da gestação, quando a insulino resistência é máxima. Objetivo: Descrever critérios diagnósticos para DMG mais utilizados por médicos ginecologistas e obstetras em atendimento ambulatorial às gestantes residentes em Araguari-MG. Trata-se de um estudo descritivo e exploratório analítico, observacional e de levantamento, por questionário direto, comparando distintos critérios diagnósticos para DMG, utilizados ambulatorialmente. Os dados foram armazenados, tratados e organizados descritivamente em um banco de dados do Microsoft Office Excel ${ }^{\circledR}$. Para uma provável análise inferencial, utilizou-se teste $\mathrm{G}$ com o auxílio do software BioEstat5.3. Dos entrevistados, a maioria são mulheres especializadas a menos de 20 anos em universidades públicas de Minas Gerais, dentre os quais, a maioria se considera atualizado, porém não utilizam predominantemente o critério do Ministério da Saúde para diagnostico de DMG. O estudo observou uma concordância com o padrão nacional da realidade médica atual, que evidencia uma feminilização dos profissionais ginecologistas e obstetras.
\end{abstract}

Palavras-chave: Diabetes Mellitus Gestacional; Diabetes Mellitus; Critérios; Diagnóstico.

\begin{abstract}
Gestational diabetes (GDM) is defined as any degree of impaired glucose tolerance, resulting in hyperglycemia of varying severity, which detection occurred during pregnancy. GDM is a disorder of the second half of pregnancy, when insulin resistance is maximum. Describe diagnostic criteria for gestational diabetes most frequently used by medical gynecologists and obstetricians in outpatient care for pregnant women living in Araguari-MG. This is a descriptive and exploratory analytical, observational and survey study, using a direct questionnaire, comparing different diagnostic criteria for GDM, used on an outpatient basis. The data were stored, processed, and descriptively organized in a Microsoft Office Exce $^{\circledR}$ database. For a probable inferential analysis, a $\mathrm{G}$ test was used with the aid of the BioEstat5.3 software. Of the interviewees, the majority are specialized women less than 20 years old in public universities in Minas Gerais, among whom, the majority consider themselves updated, but do not predominantly use the criteria of the Ministry of Health for diagnosing GDM. The study observed an agreement with the national standard of the current medical reality, which shows a feminization of gynecologists and obstetricians. However, aspects related to the diagnosis of GDM require a professionals' more optimized approach.
\end{abstract}

Keywords: $\quad$ Gestational Diabetes Mellitus; Diabetes Mellitus; Criteria; Diagnostic. 


\section{INTRODUÇÃO}

O diabetes gestacional é definido como uma redução da tolerância à glicose, resultando em hiperglicemia de gravidade variável, cujo início ou detecção ocorre durante a gravidez (MASSUCATI et al., 2012). Sua fisiopatologia é explicada pela elevação de hormônios contrarreguladores da insulina, pelo estresse fisiológico imposto pela gravidez e por fatores predeterminantes (genéticos ou ambientais). 0 principal hormônio relacionado com a resistência à insulina durante a gravidez é o lactogênico placentário, contudo, sabe-se hoje que outros hormônios hiperglicemiantes como cortisol, estrógeno, progesterona e prolactina também estão envolvidos (AMERICAN DIABETES ASSOCIATION et al., 2010).

Na mulher não diabética a gravidez está associada com profundas alterações no metabolismo energético. A glicose materna é a mais importante fonte de energia para o feto. Os níveis circulantes de glicose, aminoácidos, ácidos graxos livres, cetonas e triglicerídeos estão elevados, ao passo que a secreção de insulina, em resposta à glicose, está aumentando (RANCIARO; MAUAD, 2006).

Os níveis de estrógeno e progesterona produzidos pela placenta aumentam na gestação e são responsáveis, em parte, pelas alterações do metabolismo glicídico materno. Desse modo, o estrógeno e a progesterona agem como antagônicos à insulina, diminuindo sua eficácia nos tecidos periféricos. O cortisol aumenta no final da gestação e atua reduzindo a sensibilidade tecidual à insulina, assim, eleva os níveis glicêmicos pelo estímulo a gliconeogênese dos aminoácidos e antagoniza a ação da insulina no músculo e no tecido adiposo (SAUNDERS; PADILHA, 2009).

Em 2010, a Associação Internacional de Grupos de Estudos em Diabetes e Gravidez (IADPSG), um grupo de consenso internacional com representantes de várias organizações obstétricas e de diabetes, incluindo a American Diabetes Association (ADA), recomendou uma alteração nesta terminologia. Atualmente, o diabetes diagnosticado durante a gravidez pode ser classificado como pré-gestacional ou gestacional (INTERNATIONAL ASSOCIATION OF DIABETES AND PREGNANCY STUDY GROUPS CONSENSUS PANEL et al., 2010)
O DMG é caracteristicamente um distúrbio da segunda metade da gestação, quando a insulinoresistência é máxima. Enquanto, a hiperglicemia identificada no primeiro trimestre denota, quase sempre, doença pré-gestacional (NOGUEIRA, 2011). Aproximadamente $7 \%$ das gestações são complicadas pelo DMG, resultando em mais de 200 mil casos por ano. A prevalência pode variar de 1 a $14 \%$ de todas as gestações, dependendo da população estudada e dos testes de diagnóstico empregados. (AMERICAN DIABETES ASSOCIATION et al., 2010)

As controvérsias em critérios para diagnóstico do DMG são inúmeras, tornando difícil determinar sua real prevalência. Esta varia conforme os critérios diagnósticos, o país, a instituição, os fatores ambientais e a população sob estudo. A Sociedade Brasileira de Diabetes (SBD), mesmo com as limitações apresentadas, sugere a utilização dos novos critérios internacionais, pois são os únicos determinados por estudo que demonstrou associação entre os valores da glicemia materna e os desfechos perinatais (SOCIEDADE BRASILEIRA DE DIABETES et al., 2015).

Desta forma, os profissionais de saúde, em especial os obstetras, devem se atualizar constantemente com relação aos critérios diagnósticos de diabetes mellitus gestacional, levando em consideração as recomendações tanto da OMS (Organização Mundial de Saúde) como da SBD, ADA e Federação Brasileira das Associações de Ginecologia e Obstetrícia (FEBRASGO), durante o pré-natal, de modo a unificar os critérios e rastrear todos os possíveis casos de maneira uniforme. Assim, o presente estudo teve como objetivo descrever critérios diagnósticos mais utilizados para diabetes gestacional e fontes utilizadas para atualização entre os profissionais médicos ginecologistas e obstetras atuantes no município de Araguari-MG.

\section{MÉTODOS}

Trata-se de um estudo transversal, descritivo, de cunho quantitativo. $O$ presente estudo é classificado como analítico, observacional e de levantamento, por meio de questionário direto. Esse tem como fator avaliativo dados de diagnósticos para diabetes gestacional utilizados por profissionais em atendimento ambulatorial do serviço público de 
Araguari/MG. A pesquisa foi aprovada pelo Comitê de Ética sob o no $3.031 .885 / 2018$.

A população deste estudo contou com os 10 médicos com título de especialista em Ginecologia e Obstetrícia, que trabalham no Centro Ambulatorial Doutor Romes Nader e/ou nas Unidades Básicas de Saúde do Município de Araguari/MG.

Aplicou-se um questionário fechado, contendo 20 questões sobre idade, sexo, ano de formatura, instituição de graduação em Medicina, ano de conclusão da residência em Ginecologia e Obstetrícia (G.O.), instituição de realização da residência em G.O., serviços de saúde nos quais realizam atendimento e os métodos diagnósticos utilizados para detecção do diabetes gestacional.

As alternativas para respostas às perguntas do questionário embasaram-se nos critérios adotados pelo Ministério da Saúde, referências da Federação Brasileira das Associações de Ginecologia e Obstetrícia (FEBRASGO), referências ligadas a centros acadêmicos e pesquisa, como o HAPO, e da OMS. Dentre estes, o profissional deveria optar pelo mais utilizado em sua prática diária. Para armazenamento e cálculo descritivo utilizou-se o Microsoft Office Exce ${ }^{\circledR}$ e para análise inferencial dos dados, o Teste $G$ do software BioEstat5.3.

\section{RESULTADOS}

Todos os 10 médicos especialistas em Ginecologia e Obstetrícia que trabalham na rede pública do município de Araguari-MG aceitaram participar do estudo e foram entrevistados.

Dos profissionais atuantes $70 \%$ são do sexo feminino e a maioria com idade entre 20 e 39 anos (50\%). Em relação ao ano de conclusão do curso de Medicina, $50 \%$ se formaram entre $2000-2009$, 30\% entre 2010 2019 e $20 \%$ entre $1970-1979$, sendo que, $60 \%$ deles se formaram em universidades públicas e $40 \%$ em instituições privadas.

Quanto à especialização em Ginecologia e Obstetrícia $50 \%$ dos profissionais entrevistados concluíram essa formação nos últimos nove anos, ou seja, entre os anos de 2010 e 2019 sendo que a maioria realizada em instituições públicas (90\%) (Tabela 1).

Tabela 1 - Perfil socioeducativo dos profissionais ginecologistas obstetras atuantes no município de Araguari- MG; 2019.

\begin{tabular}{|c|c|c|c|}
\hline & Variáveis & $n$ & $\%$ \\
\hline \multirow{2}{*}{ Sexo } & Masculino & 3 & $30 \%$ \\
\hline & Feminino & 7 & $70 \%$ \\
\hline \multirow{5}{*}{ Idade } & $20-29$ & 2 & $20 \%$ \\
\hline & $30-39$ & 3 & $30 \%$ \\
\hline & $40-49$ & 3 & $30 \%$ \\
\hline & $50-59$ & 0 & $0 \%$ \\
\hline & $60-69$ & 2 & $20 \%$ \\
\hline \multirow{5}{*}{ Ano de Formatura } & 1970-1979 & 2 & $20 \%$ \\
\hline & 1980-1989 & 0 & $0 \%$ \\
\hline & 1990-1999 & 0 & $0 \%$ \\
\hline & 2000-2009 & 5 & $50 \%$ \\
\hline & 2010-2019 & 3 & $30 \%$ \\
\hline \multirow{2}{*}{$\begin{array}{l}\text { Tipo de Instituição de } \\
\text { Formação }\end{array}$} & Pública & 6 & $60 \%$ \\
\hline & Privada & 4 & $40 \%$ \\
\hline \multirow{5}{*}{$\begin{array}{l}\text { Ano de conclusão da } \\
\text { Especialização (G.O.) }\end{array}$} & 1970-1979 & 2 & $20 \%$ \\
\hline & 1980-1989 & 0 & $0 \%$ \\
\hline & 1990-1999 & 0 & $0 \%$ \\
\hline & 2000-2009 & 3 & $30 \%$ \\
\hline & 2010-2019 & 5 & $50 \%$ \\
\hline \multirow{2}{*}{$\begin{array}{l}\text { Tipo de Instituição de } \\
\text { Especialização }\end{array}$} & Pública & 10 & $100 \%$ \\
\hline & Privada & 0 & $0 \%$ \\
\hline \multirow{3}{*}{ Inst. Especialização } & Univ. Federal de Uberlândia (UFU) & 7 & $70 \%$ \\
\hline & Univ. Federal do Triângulo Mineiro (UFTM) & 2 & $20 \%$ \\
\hline & Hospital de Base do Distrito Federal (HBDF) & 1 & $10 \%$ \\
\hline
\end{tabular}


Quanto aos critérios utilizados para diagnóstico de Diabetes Mellitus Gestacional (DMG) constatou-se que a maioria (80\%) utiliza preferencialmente a glicemia casual maior ou igual a $92 \mathrm{mg} / \mathrm{dL}$, mas menor que $126 \mathrm{mg} / \mathrm{dL}$ na primeira visita pré-natal ou pelo menos um resultado anormal no teste de tolerância oral à glicose (glicemia de jejum, uma e duas horas após ingestão oral de 75 gramas de dextrosol) realizado entre 24 e 28 semanas de gestação.

Ter glicemia de jejum maior ou igual a $126 \mathrm{mg} / \mathrm{dL}$, foi o critério escolhido por $10 \%$ dos entrevistados. E, hemoglobina glicada maior ou igual a 6,5\% também foi a opção diagnóstica de $10 \%$ dos profissionais.

Os demais critérios abordados, (1. glicemia casual maior ou igual a $200 \mathrm{mg} / \mathrm{dL}$, que é posteriormente confirmada pela glicemia de jejum ou hemoglobina glicada; 2. glicemia em jejum maior ou igual a $126 \mathrm{mg}$ $\%$ em duas medidas ou medidas aleatórias maior ou igual a 200mg \%; 3. glicemia plasmática de jejum maior ou igual a $85 \mathrm{mg} / \mathrm{dL}$ ) não foram relatados como utilizados para diagnóstico de DMG para os profissionais do município estudado.

Em relação a percepção de atualização médica, a maioria dos profissionais entrevistados (70\%) se consideram atualizados ou parcialmente atualizados (30\%). Essa busca de conhecimento advém em $40 \%$ da Federação Brasileira das Associações de Ginecologia e Obstetrícia (FEBRASGO) e $40 \%$ de fontes internacionais (Tabela 2). Uma minoria dos entrevistados, relatou buscar conhecimento pelo Ministério da Saúde (10\%) e por meio de congressos (10\%). Tal achado é significativo, tendo em vista que todos os profissionais entrevistados trabalham para o Ministério da Saúde, via atendimentos no Sistema Único de Saúde (SUS), mas a grande maioria (90\%) não utiliza preferencialmente fontes desse Ministério como meio de atualização

Tabela 2 - Relação das fontes de atualização com o ano de formatura dos ginecologistas obstetras atuantes no município de Araguari- MG; 2019.

\begin{tabular}{lccccc}
\hline \multicolumn{1}{c}{ Fontes de Atualização } & \multicolumn{2}{c}{ Ano de formatura } & \multicolumn{2}{c}{ Porcentagem } \\
\cline { 2 - 6 } & $1970-1979$ & $1980-1999$ & $2000-2009$ & $2010-2019$ & $\%$ \\
Ministério da Saúde (SUS) & 0 & 0 & 1 & 0 & $10 \%$ \\
FEBRASGO & 1 & 0 & 2 & 1 & $40 \%$ \\
Referências Internacionais & 1 & 0 & 1 & 2 & $40 \%$ \\
Referências ligadas a algum centro acadêmico & 0 & 0 & 0 & 0 & $0 \%$ \\
Congressos & 0 & 0 & 1 & 0 & $10 \%$ \\
\hline
\end{tabular}

Fonte: os autores

\section{DISCUSSÃO}

Os dados socioeducacionais encontrados no presente estudo foram comparados com os dados do estudo Demografia Médica no Brasil (2018), que traz um panorama nacional dos Ginecologistas e Obstetras. Enquanto a média de idade averiguada pela presente pesquisa foi de 41,9 anos, nota-se que os profissionais de Araguari-MG são aproximadamente 19 anos mais jovens do que a média nacional de idade dos Ginecologistas e Obstetras, que é de 59,6 anos (No Brasil, Demografia Médica et al., 2018). Todavia, é válido ressaltar que esses 19 anos de diferença não representam tamanha discrepância, tendo em vista que a tendência nacional é de que os médicos se tornem especialistas cada vez mais jovens, diminuindo a média de idade dos Ginecologistas e Obstetras no Brasil.

Quanto ao sexo, o estudo em questão apresentou $70 \%$ de mulheres, enquanto a porcentagem nacional de Ginecologistas Obstetras do sexo feminino é de $56,6 \%$ (No Brasil, Demografia Médica et al., 2018). Logo, percebe-se consonância entre os dados nacionais e municipais, tendo em vista que ambos apontam a prevalência do sexo feminino na especialidade pesquisada. Outro dado de conformidade com o levantamento Demografia Médica no Brasil (2018), foi referente ao tipo de 
instituição de formação, em ambos os estudos, a maioria dos especialistas ainda é oriunda de instituições públicas.

Quanto aos critérios mais utilizados para diagnóstico do Diabetes Mellitus gestacional, o estudo de Zapelini et al. (2015) preconizou a referência da OMS, assemelhando-se ao encontrado no presente estudo, no qual $80 \%$ dos ginecologistas e obstetras entrevistados na pesquisa escolheram glicemia de jejum maior ou igual a $92 \mathrm{mg} / \mathrm{dL}$, mas menor que 126 $\mathrm{mg} / \mathrm{dL}$ na primeira visita pré-natal, ou pelo menos um resultado anormal no teste de tolerância oral à glicose (glicemia de jejum, uma e duas horas após ingestão oral de 75 gramas de dextrosol) realizado entre 24 e 28 semanas de gestação. Assim como estudo realizado por Da Silva et al. (2017), cujo diagnóstico de DMG também foi feito com base no teste de tolerância oral à glicose.

De acordo com Costa et al. (2006), em um estudo de Prevalência de Diabetes Mellitus em Pelotas-RS, para comparar a prevalência de Diabetes Mellitus em população adulta, residente em área urbana, foi adotado como ponto de corte da pesquisa, glicemia de jejum para reconhecimento de diabetes maior ou igual a $126 \mathrm{mg} / \mathrm{dl}$ e menor que $140 \mathrm{mg} / \mathrm{dl}$. Concomitantemente ao presente estudo, em que ter glicemia de jejum maior ou igual a $126 \mathrm{mg} / \mathrm{dL}$, foi o critério escolhido por $10 \%$ dos entrevistados (WORLD HEALTH ORGANIZATION et al., 1965).

Segundo Andriolo et al. (2009), manter o nível de hemoglobina glicada (A1C) inferior a 7\% é uma das principais metas para o bom controle da doença e para evitar complicações crônicas. Entretanto 10\% dos profissionais que participaram da atual pesquisa utilizaram esse método de controle como opção diagnóstica.

\section{CONCLUSÃO}

Relativamente aos dados socioeducacionais observados neste estudo, é possível afirmar que eles estão em concordância com o padrão nacional vigente. Quanto aos critérios diagnósticos de DMG, evidencia-se a necessidade de buscar estratégias de prática, ensino e aprendizado mais padronizadas e sintonizadas com as condições e necessidades atuais e tendências futuras, baseadas na melhor evidência científica disponível. De modo que, nas consultas públicas de pré-natal, possa ocorrer o diagnóstico precoce do DMG e diminuição das complicações oriundas de diagnósticos tardios dessa patologia.

\section{REFERÊNCIAS}

AMERICAN DIABETES ASSOCIATION et al. Standards of medical care in diabetes - 2010. Diabetes care, $v$. 33, n. Supplement 1, p. S11-S61, 2010.

COSTA, Juvenal Soares Dias da et al. Prevalência de Diabetes Mellitus em Pelotas, RS: um estudo de base populacional. Revista de Saúde Pública, v. 40, n. 3, p. 542-545, 2006.

DA SILVA, Amanda L. et al. Neonatal outcomes according to different therapies for gestational diabetes mellitus. Jornal de pediatria, v. 93, n. 1, p. 87-93, 2017.

\section{INTERNATIONAL ASSOCIATION OF DIABETES AND} PREGNANCY STUDY GROUPS CONSENSUS PANEL et al. International association of diabetes and pregnancy study groups recommendations on the diagnosis and classification of hyperglycemia in pregnancy. Diabetes care, v. 33, n. 3, p. 676-682, 2010.

MASSUCATTI, Lais Angelo et al. Prevalência de diabetes gestacional em Unidades de Saúde Básica. Revista de enfermagem e atencao a saúde, v. $1, \mathrm{n}$. 01, 2012.

NETTO, Augusto Pimazoni et al. Atualização sobre hemoglobina glicada (HbA1C) para avaliação do controle glicêmico e para o diagnóstico do diabetes: aspectos clínicos e laboratoriais. Jornal Brasileiro de Patologia e Medicina Laboratorial, v. 45, n. 1, p. 3148, 2009.

NO BRASIL, Demografia Médica. Coordenação de Mário Scheffer; equipe de pesquisa: Alex Cassenote, Aline Gil Alves Guilloux, Aureliano Biancarelli, Bruno Alonso Miotto e Giulia Marcelino Mainardi. São Paulo: Departamento de Medicina Preventiva da Faculdade de Medicina da USP, 2018.

NOGUEIRA, Anelise Impeliziere et al. Diabetes Gestacional: perfil e evolução de um grupo de pacientes do Hospital das Clínicas da UFMG. Revista Médica de Minas Gerais, v. 21, n. 1, p. 32-41, 2011. 
RANCIARO, Rose Mary de Castro; MAUAD-FILHO, Francisco. Efeitos da ingestão de glicose sobre a circulação materno-fetal. Revista Brasileira de Ginecologia e Obstetrícia, v. 28, n. 12, p. 693-699, 2006.

SAUNDERS, C.; PADILHA, P. C. Diabetes na gestação. Accioly E, Saunders C, Lacerda E. Nutrição em obstetrícia e pediatria. Rio de Janeiro: Cultura Médica, p. 191-207, 2009.

SOCIEDADE BRASILEIRA DE DIABETES. Diabetes mellitus gestacional: diagnóstico, tratamento e acompanhamento pós-gestação. Diretrizes SBD. 2014-2015

WORLD HEALTH ORGANIZATION et al. Diabetes mellitus: report of a WHO Expert Committee [meeting held in Geneva from 24 to 30 November 1964]. 1965.

ZAPELINI, Raphaela Mazon et al. Critérios diagnósticos e prevalência de Diabetes Mellitus Gestacional em um hospital do sul de Santa Catarina.

Revista AMRIGS, v. 59, n. 3, p. 177-181, 2015. 